\title{
Ultrafast Thermal Melting in Nonequilibrium Warm Dense Copper
}

\author{
N. Jourdain, ${ }^{1,2}$ L. Lecherbourg, ${ }^{2,3}$ V. Recoules, ${ }^{2}$ P. Renaudin, ${ }^{2}$ and F. Dorchies $\circledast^{1, *}$ \\ ${ }^{1}$ Univ. Bordeaux, CNRS, CEA, CELIA (Centre Lasers Intenses et Applications), UMR 5107, F-33400 Talence, France \\ ${ }^{2}$ CEA, DAM, DIF, F-91297 Arpajon, France \\ ${ }^{3}$ LOA, ENSTA ParisTech, CNRS, Ecole Polytechnique, Univ. Paris-Saclay, 91120 Palaiseau, France
}

(Received 24 April 2020; revised 13 January 2021; accepted 25 January 2021; published 12 February 2021)

\begin{abstract}
The ultrafast dynamics of the loss of crystalline periodicity is investigated in femtosecond laser heated warm dense copper, by the original use of x-ray absorption near-edge specific structures just above the $L 3$ edge. The characteristic time is observed near $1 \mathrm{ps}$, for specific energy density ranging from 1 to $5 \mathrm{MJ} / \mathrm{kg}$, using ps-resolution x-ray absorption spectroscopy. The overall experimental data are well reproduced with two-temperature hydrodynamic simulations, supporting a thermal phase transition.
\end{abstract}

DOI: 10.1103/PhysRevLett.126.065001

Since the advent of intense and femtosecond laser pulses [1], the study of their interaction with matter has raised a strong enthusiasm. Among other uses, it is a privileged way to bring matter in extreme states of temperature and pressure, with fundamental implications in various fields of physics including laser micromachining, planetology, and inertial confinement fusion [2-5]. Despite these major scientific motivations, the description of this so-called warm dense matter (WDM) is still highly challenging, due to partial disorder, correlated ions, electron degeneracy, and strong coupling between electron structure and ion bonding [6]. As femtosecond laser pulses lead to conditions far from equilibrium (hot electrons and still cold lattice), they offer a unique opportunity to get deeper understanding of the electron-ion dynamic interplay $[7,8]$.

There has been considerable theoretical interest in the dynamics of phase transitions in these nonequilibrium situations [9-11]. In covalent materials, electronically driven phase transitions were reported, also referred to as nonthermal melting. They are attributed to changes in the potential-energy landscape of the lattice by the excited electrons [12-15]. In metals, a thermal process is generally considered driven by the progressive energy transfer from the electrons to the lattice $[4,16-18]$. Theoretical calculations predict different behaviors depending on the nature of the electronic density of state $[10,19]$. But even for a given element (e.g., gold as a full $d$-band metal prototype) the question is still debated whether the strongly excited electron system can cause lattice strengthening [10], or weakening [20], also referred to as bond hardening or softening, respectively. There is therefore a critical need for reliable experimental data to constrain the different theoretical approaches, especially for such full $d$-band metals.

Understanding of the electron-ion dynamic interplay requires addressing two major questions. The first difficulty consists of controlling the energy exchange between electrons and ions. The two-temperature model (TTM) has been proposed to provide a simple view of the dynamical process, assuming that both subsystems can be described by respective temperatures $T_{e}$ and $T_{i}$ [21]. Their evolution depends on physical parameters (heat capacities $C_{e}$ and $C_{i}$, and electron-ion coupling factor $G$ ) that have a strong impact on the structural dynamics of the system and lead whether any phase transition occurs at higher or lower ion temperature rather than the one expected at thermal equilibrium [22]. This is the reason why $C_{e}, C_{i}$ and $G$ must be accurately calculated as a function of the temperature.

The second challenge is to get time-resolved data measurements directly related to the phase transition dynamics. That requires appropriate diagnostics whose implementation is complicated by the fact that the sample is generally destroyed after each laser shot. The first studies were carried out with femtosecond all-optical pump-probe experiments [23-25]. They have provided significant advances to the field, including the observation of a quasisteady state lasting about $10 \mathrm{ps}$ in warm dense gold [18,26-28]. However, the link between the phase transitions and the associated changes in the amplitude and phase of an optical probe remains indirect. Time-resolved diffraction experiments of $\mathrm{x}$ rays $[13,14]$ and electrons $[4,16,17]$ have also been reported. These constitute real technological prowess and give access to the temporal evolution of the atomic structure. In the regime of high specific energy density ( $\geq 1 \mathrm{MJ} / \mathrm{kg}$ ), homogeneous melting transition has been observed to be complete within a relatively long time of $\sim 10 \mathrm{ps}$ in warm dense gold $[4,17]$, supporting the theoretical predictions of an increased and $T_{e}$-dependent melting temperature [10,29].

$\mathrm{X}$-ray absorption near-edge spectroscopy (XANES) has been recently proposed to probe nonequilibrium warm dense matter [7], demonstrating time resolution down to the femtosecond [30-32]. This alternative diagnostic has great potential because it can provide simultaneous information 
at the atomic scale on the structures of electrons and nuclei. The electron temperature evolution has been retrieved in warm dense copper and supports the TTM predictions with $T_{e}$ dependent $C_{e}$ and $G[30,33,34]$. The short-range picosecond disordering has been resolved in warm dense aluminum [35]. This last provided data on the ion temperature dynamics, but not on the phase transition from solid to liquid in which the short-range order is not significantly changed [36].

In this Letter, we demonstrate that time-resolved XANES can be exploited to resolve the ultrafast dynamics of the phase transition from fcc solid to aperiodic warm dense copper. In addition to the original method presented to track the loss of the crystal periodicity, the diagnostic has already been proven to provide simultaneous control of the $T_{e}$ dynamics. This results in invaluable data on the solid-toliquid transition. Such transition is considered from the point of view of the electronic structure that drives most of the macroscopic physical properties. In the range from 1 to $5 \mathrm{MJ} / \mathrm{kg}$, this melting time is observed near one picosecond. It compares quite well with simple TTM predictions using $T_{e}$-dependent $C_{e}$ and $G$, and considering the melting temperature at thermal equilibrium.

In a crystalline solid, the electronic structure is intimately related to the periodicity of the atomic lattice. Figure 1(a) shows such electronic band structure in the reciprocal space for fcc-Cu. Extrema in the dispersion relation $(d E / d k=0)$ lead to band edges in the density of state [Fig. 1(b)], which are referred to as van Hove singularities [37]. These result in corresponding peaks in the $\mathrm{x}$-ray absorption spectrum [in the $10 \mathrm{eV}$ above the $\mathrm{Cu} L$ edge as reported in Fig. 1(c)]. They are characteristic of the crystalline phase. For example, a peak located at another energy is observed in bcc-Cu [38]. Note that this signature of the crystalline phase is different from the features that result from the photoelectron wave function scattering on the nearestneighboring atoms and that are related to the short-range atomic order, such as sharp resonant lines or EXAFS oscillations [39].

When the solid copper turns to liquid, the lattice periodicity is lost and the consequent peaks above the $\mathrm{x}$-ray absorption $L 3$ edge are expected to vanish. Ab initio molecular dynamic simulations (AIMD) have been performed in order to confirm this intuitive view in warm dense copper. The assumptions and methodology are fully detailed in Ref. [40]. Some calculations are reported for illustration in Figs. 2(b) and 2(c). As it was discussed and exploited in previous papers, a pre-edge is observed as the electron temperature $T_{e}$ increases [30,33,34]. It is partially compensated by the reduction of the absorption just at the L3 edge (peak labeled "1"). They both result from the promotion of some electrons from the $3 d$ band up to higher energy states when $T_{e}$ increases. When $T_{i}$ increases, the postedge peaks (labeled "2" and "3") disappear, as a direct consequence of the van Hove singularities vanishing.

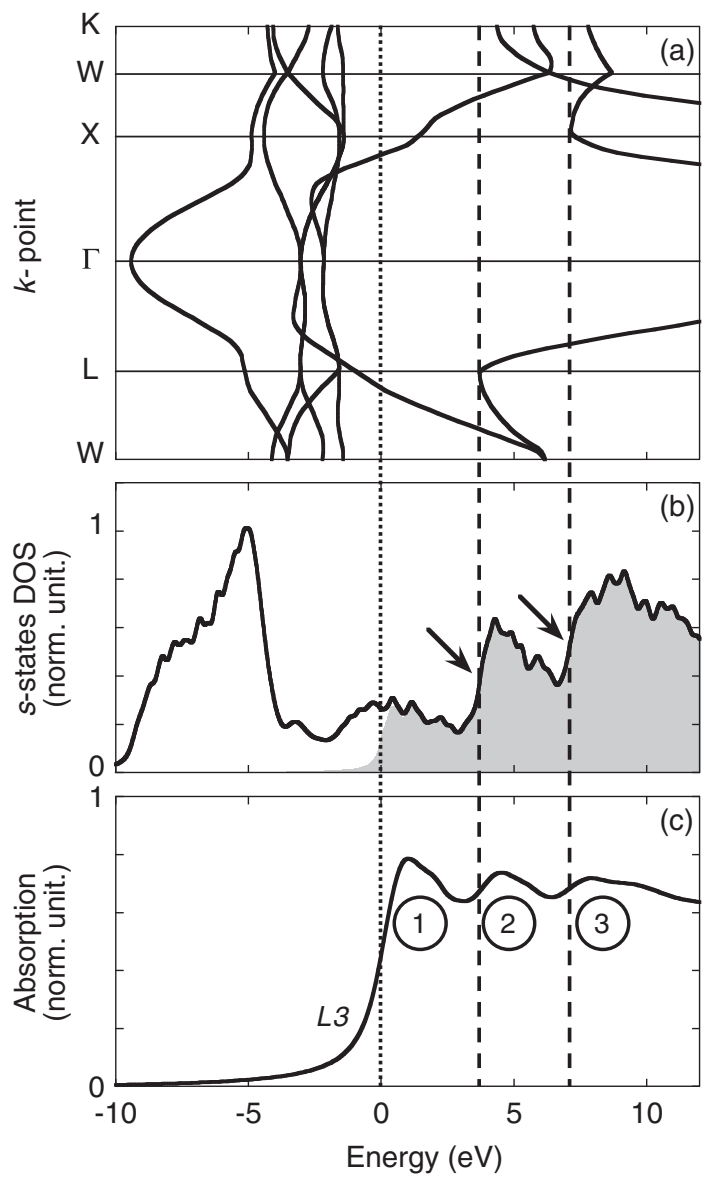

FIG. 1. Principle of the van Hove singularities in fcc-Cu and their signature on the L3-edge XANES spectrum. (a) Electronic band structure. (b) Corresponding projected DOS on $s$ states. The shaded area (gray) represents the unoccupied states. The $k$ points ( $L$ and $X$ ) where the energy reaches extrema (vertical dashed lines) lead to singularities in the DOS (edges marked by arrows). (c) They result in peaks labeled 2 and 3 in the XANES spectrum. The peak labelled 1 is the consequence of the Fermi energy $E_{F}$ that separates occupied and unoccupied states. The $x$-axis origin (dotted vertical line) is set to $E_{F}$ in (a) and (b), and to the associated L3-edge energy $(932.5 \mathrm{eV})$ in (c).

Time-resolved XANES spectra near the $\mathrm{Cu} L 3$ edge have been recorded on a dedicated tabletop setup developed at CELIA laboratory, and fully described in Ref. [41]. The laser-based x-ray source is generated by the interaction of a probe beam (600 fs FWHM, 80 mJ) with xenon clusters, providing about $35000 \mathrm{x}$-ray photons per $\mathrm{eV}$ per pulse with $1.2 \pm 0.2 \mathrm{ps}$ rms duration at the sample plane [42] Samples are made of $80 \pm 5 \mathrm{~nm}$ of $\mathrm{Cu}$ deposited on $0.9 \mu \mathrm{m}$ of Mylar. The copper thickness was chosen to expect a homogeneous energy deposition limited to copper (optical penetration depth of $12.7 \mathrm{~nm}$, and ballistic electron mean free path of $70 \mathrm{~nm}$ [30]). Sample are heated by $p$-polarized optical pulses of $300 \mathrm{fs}$ duration, at $30^{\circ}$ incidence. The spatial profile is flat top on the sample, with a diameter $(\sim 1 \mathrm{~mm})$ larger than the $\mathrm{x}$-ray spot 

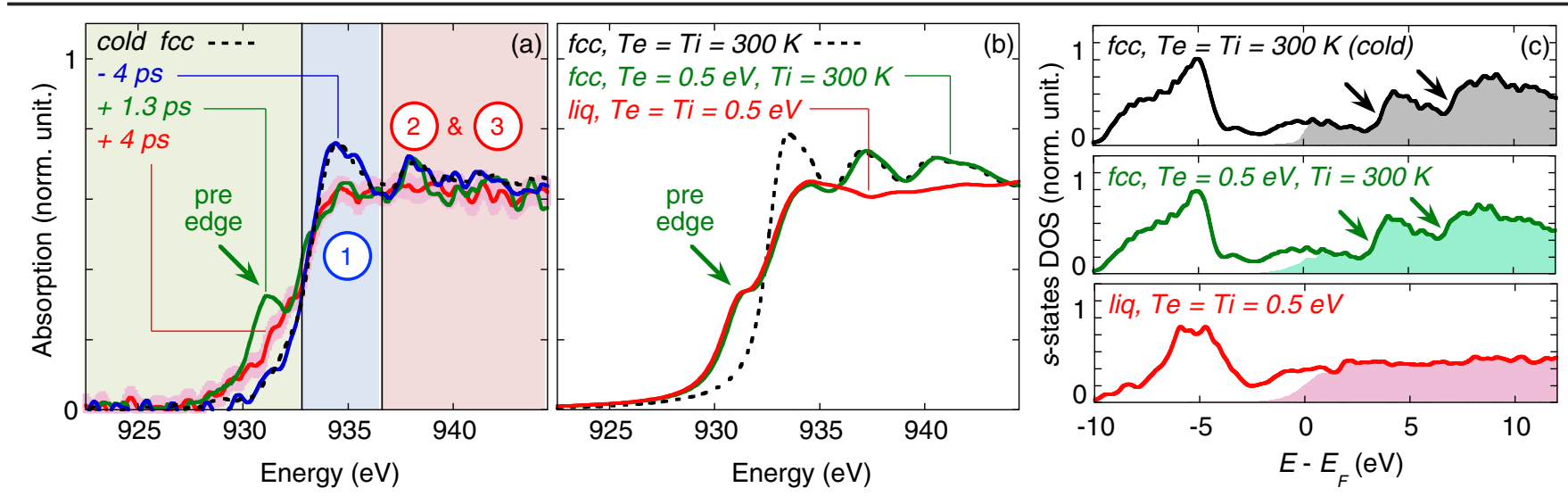

FIG. 2. (a) Some time-resolved XANES spectra measured at $F_{\text {abs }}=0.065 \pm 0.015 \mathrm{~J} / \mathrm{cm}^{2}$. For clarity reason, the error bar is only indicated for the delay +4 ps. The energy intervals for spectral integration of the pre-edge and the postedge peaks labeled 1, then 2 and 3 are indicated with shaded color areas (respectively, in green, blue, and red). (b) Some calculated XANES spectra. (c) Corresponding computed projected DOS on $s$ states. The van Hove singularities characteristic of the fcc crystalline phase are indicated with arrows.

(gaussian profile with $300 \mu \mathrm{m}$ FWHM diameter). The laser energy reaches $\sim 10 \mathrm{~mJ}$, corresponding to incident fluence up to $\sim 1 \mathrm{~J} / \mathrm{cm}^{2}$. As the heated area is usually destroyed after a single shot, the sample is replaced by the use of a motorized translation. The effective repetition rate is $2 \mathrm{~Hz}$.

Some registered spectra are presented in Fig. 2(a) for an absorbed laser fluence $F_{\text {abs }}=0.065 \pm 0.015 \mathrm{~J} / \mathrm{cm}^{2}$ $(\sim 1 \mathrm{MJ} / \mathrm{kg})$. Each results from the accumulation of more than 2000 laser shots, in order to obtain a signal-to-noise ratio sufficient to resolve the tenuous postedge peaks. Just after the laser heating (delay $1.3 \mathrm{ps}$ ), a pre-edge is observed, testifying to the $T_{e}$ increase. The peaks 2 and 3 are still clearly observed, demonstrating a strong nonequilibrium situation with hot electrons and still fcc-Cu lattice. These peaks then disappear at $4 \mathrm{ps}$, which reveals an ultrafast phase transition from fcc-solid to liquid. The evolutions of the peak 1, and of the peaks 2 and 3 are plotted in Fig. 3 as a

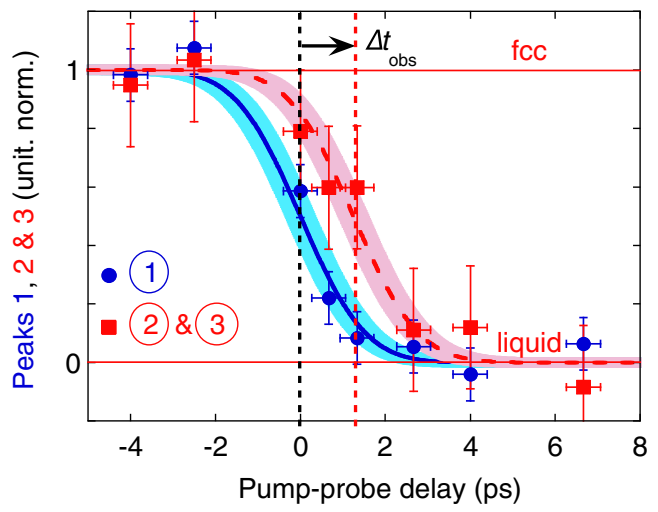

FIG. 3. Time evolution of the peak 1 (blue circles), and the peaks 2 and 3 (red squares) that sign the transition of the electron structure from fcc solid (value set to 1 ) to liquid (value set to 0 ). The laser fluence is the same as in Fig. 2(a). The instrumental response is reported (full line in blue). The data of the peaks 2 and 3 are fitted with the same function, but shifted by a delay $\Delta t_{\mathrm{obs}}$ (dashed line in red). function of the pump-probe delay. They are evaluated by spectral integration [intervals indicated in Fig. 2(a)]. Normalized units, resulting from comparison with cold fcc and liquid spectra are chosen to compare them more easily (see the Supplemental Material [43] for details on the normalization procedure). As expected, the evolution of the peak 1 is simultaneous with the laser heating (zero delay). The decreasing time is limited by the convolution by the $1.2 \pm 0.2 \mathrm{ps}$ rms Gaussian x-ray pulse duration. The corresponding instrumental response is plotted in Fig. 3 (full line). The evolution of the peaks 2 and 3 presents the same decreasing time limited by the instrumental response. But the whole set of data is time shifted by $\Delta t_{\text {obs. }}$. This observation suggests a sudden phase transition, that starts at $\Delta t_{\mathrm{obs}}$ after the electrons heating. At the relatively low fluence considered here, this measured melting time is estimated at $\Delta t_{\mathrm{obs}}=1.25 \pm 0.5 \mathrm{ps}$ (the fitting procedure is detailed in the Supplemental Material [43]).

As previously published, the evolution of $T_{e}$ can be retrieved from the spectral integration of the pre-edge $[30,33,34]$. Results are plotted in the Fig. 4. They are compared with the $T_{e}$ and $T_{i}$ dynamics calculated with the one-dimensional Lagrangian hydrodynamic code ESTHER in which the TTM is consistently integrated [44]. The laser absorbed fluence measured in the experiment is used. $T_{e^{-}}$ dependent coefficients are considered for the electron heat capacity $C_{e}$ and the electron-ion coupling factor $G$ [22]. The ion heat capacity is derived from the Bushman Lomonosov Fortov (BLF) equations of state that are used in ESTHER [45]. A satisfactory agreement is observed for $T_{e}$ time evolution, giving a high degree of confidence in the control of the temperatures dynamics. Under these rather simple considerations, we sought to compare the $\Delta t_{\text {obs }}$ measurement with the time $\Delta t_{m}$ needed for $T_{i}$ to pass over the melting temperature.

The delay $\Delta t_{\text {obs }}$ for the loss of lattice periodicity has been measured at different absorbed laser fluences, by using the 


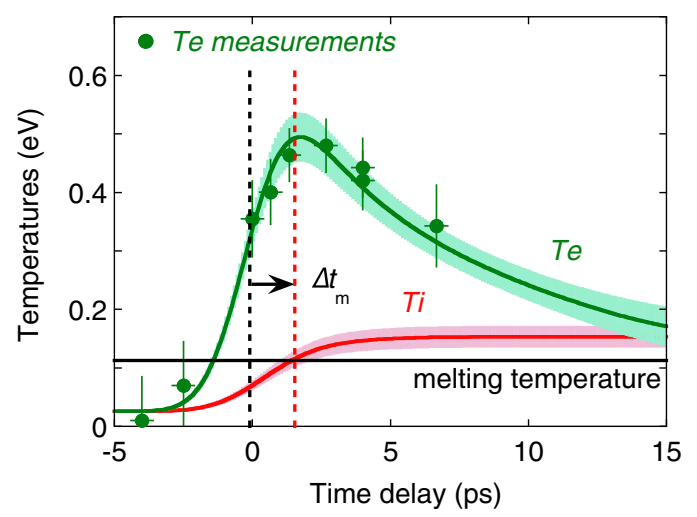

FIG. 4. Time evolution of both electron $\left(T_{e}\right)$ and ion $\left(T_{i}\right)$ temperatures estimated with the two-temperature code at $F_{\text {abs }}=$ $0.065 \pm 0.015 \mathrm{~J} / \mathrm{cm}^{2}$ (see details in the text), and convoluted with the time instrumental response. The shaded areas come from the fluence uncertainty. Values of $T_{e}$ deduced from the pre-edge measurements are reported (circles).

same procedure detailed above. Results are reported in the Fig. 5 with the corresponding error bars issued from the analysis. The specific energy density deposited in the $80 \mathrm{~nm} \mathrm{Cu}$ layer ranges from $\sim 1$ to $5 \mathrm{MJ} / \mathrm{kg}$ (corresponding to absorbed fluence from 0.065 to $0.4 \mathrm{~J} / \mathrm{cm}^{2}$ ), i.e., large enough to guarantee a homogeneous melting transition [17]. The melting times $\Delta t_{m}$ issued from ESTHER calculations are plotted for comparison. A strong mismatch is observed when considering the low temperature electronion coupling $\left(G_{0}=10^{17} \mathrm{~W} / \mathrm{m}^{3} / \mathrm{K}\right)$ and free electron gas electron heat capacity $\left(C_{e}\right.$ FEG). That illustrates the importance of properly defining these coefficients. On the other hand, the agreement is rather satisfactory when considering the coefficients $C_{e}\left(T_{e}\right)$ and $G\left(T_{e}\right)$, which have been independently constrained by the time evolution of $T_{e}$ (cf. Fig. 4 and Ref. [22]).

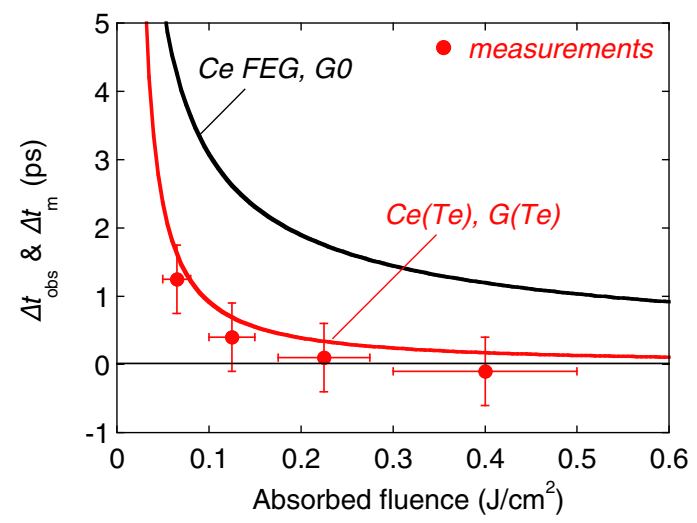

FIG. 5. Time delay $\Delta t_{\mathrm{obs}}$ for the electron structure transition from fcc to liquid, measured as a function of the absorbed laser fluence. Data are compared with two-temperature calculations performed with two sets of physical parameters $\left(\Delta t_{m}\right.$, see details in the text).
The melting temperature that is taken into account to estimate $\Delta t_{m}$ is the tabulated value at ambient conditions $T_{\text {melt }}=1358 \mathrm{~K}(0.117 \mathrm{eV})$. This has been generally considered by previously reported experimental studies $[4,17]$. Theoretical work shows that $T_{\text {melt }}$ is likely to depend on several factors, that could affect the melting time. It should increase with electronic temperature and pressure, and decrease with density during hydrodynamic expansion (see, e.g., the Refs. [46,47]). A theoretical study integrating all these mechanisms in a self-consistent way goes beyond the scope of the present experimental study, but observations seem to indicate that these effects might compensate each other.

Data indicate a melting time near $1 \mathrm{ps}$ at $1 \mathrm{MJ} / \mathrm{kg}$, and even subpicosecond up to $5 \mathrm{MJ} / \mathrm{kg}$. These values are significantly shorter than previous time-resolved electron diffraction data, measured in a similar regime in warm dense gold $[4,17]$. This contradiction, assuming that the melting is driven by the same mechanisms in full $d$-band $\mathrm{Cu}$ and $\mathrm{Au}$, is probably only apparent, since the observables are not the same. Indeed, AIMD calculations of the ion pair correlation function (see the Supplemental Material [43]) suggest that the transition of the electronic structure (probed by XANES) is more pronounced during the melting than that of the ion structure (probed by diffraction), i.e., is more sensitive to a sudden change of temperature. A last consideration concerns the time needed to reach a complete phase transition, i.e., to achieve the diffraction patterns of the liquid at equilibrium. This requires additional time needed for the solid density to relax down to the liquid one. ESTHER calculations show that following the formation of a liquid layer at the surface, the liquid-solid interface propagates inside the sample at the sound velocity ( $\sim 5 \mathrm{~km} / \mathrm{s}$ from BLF). This gives a time of $\sim 8$ ps to put the entire layer in the liquid state, a value consistent with the electron diffraction observations $[4,17]$.

In summary, we have performed a time-resolved XANES experiment to study the phase transition dynamics in femtosecond laser heated warm dense copper. Exploiting some features associated with van Hove singularities in the electron structure, the loss of the lattice periodicity is observed in the picosecond or even subpicosecond timescale, in the range of specific energy density from 1 to $5 \mathrm{MJ} / \mathrm{kg}$. A simultaneous control of the electron temperature dynamics is provided by another feature in the x-ray absorption spectra. The overall observations are fairly well reproduced by a simple two-temperature model, provided that $T_{e}$-dependent coefficients are considered [22] and assuming that the melting occurs when the ion temperature exceeds the melting temperature. Data do not indicate any bond hardening effect, which is not surprising since this effect has been predicted for $T_{e} \geq 3 \mathrm{eV}$ [10], while $T_{e}$ does not exceed $2 \mathrm{eV}$ in the present work. In order to investigate such effect, one needs to achieve higher energy deposition and to get XANES data with a femtosecond time 
resolution, which should be possible now with XFEL [48] or even with laser-based betatron $\mathrm{x}$-ray source $[32,49]$. The van Hove singularities being expected in a large number of crystalline solids, the technique reported in this paper is a priori generalizable to materials other than copper.

This work was supported by the French Agence Nationale de la Recherche, under grant OEDYP (ANR09-BLAN-0206-01), the Conseil Régional d'Aquitaine, under grants POLUX (2010-13-04-002). The authors gratefully acknowledge Anatoly Filippov, Rodrigue Bouillaud, and Laurent Merzeau for their technical assistance, and Frédéric Burgy, Clément Pégeot for laser operation. They also thank Martine Millerious from CEA and Michael Störmer from Helmholtz Zentrum Geesthacht for the samples realization. The authors thank Marc Torrent for his great help with ABINIT simulations.

*fabien.dorchies@u-bordeaux.fr

[1] D. Strickland and G. Mourou, Opt. Commun. 56, 219 (1985).

[2] T. Guillot, Science 286, 72 (1999).

[3] Q. Feng, Y. N. Picard, H. Liu, S. M. Yalisove, G. Mourou, and T. M. Pollock, Scr. Mater. 53, 511 (2005).

[4] R. Ernstorfer, M. Harb, C. T. Hebeisen, G. Sciaini, T. Dartigalongue, and R. J. D. Miller, Science 323, 1033 (2009).

[5] S. H. Glenzer et al., Science 327, 1228 (2010).

[6] National Research Council, Frontiers in High Energy Density Physics: The X-Games of Contemporary Science (National Academic Press, Washington, DC, 2003).

[7] F. Dorchies and V. Recoules, Phys. Rep. 657, 1 (2016) and references therein.

[8] B. Rethfeld, D. S. Ivanov, M. E. Garcia, and S. I. Anisimov, J. Phys. D 50, 193001 (2017).

[9] B. Rethfeld, A. Kaiser, M. Vicanek, and G. Simon, Phys. Rev. B 65, 214303 (2002).

[10] V. Recoules, J. Clérouin, G. Zérah, P. M. Anglade, and S. Mazevet, Phys. Rev. Lett. 96, 055503 (2006).

[11] E. S. Zijlstra, J. Walkenhorst, and M. E. Garcia, Phys. Rev. Lett. 101, 135701 (2008).

[12] C. W. Siders, A. Cavalleri, K. Sokolowski-Tinten, C. Tóth, T. Guo, M. Kammler, M. H. von Hoegen, K. R. Wilson, D. von der Linde, and C. P. J. Barty, Science 286, 1340 (1999).

[13] A. Rousse et al., Nature (London) 410, 65 (2001).

[14] A. M. Lindenberg et al., Science 308, 392 (2005).

[15] M. Harb, R. Ernstorfer, C. T. Hebeisen, G. Sciaini, W. Peng, T. Dartigalongue, M. A. Eriksson, M. G. Lagally, S. G. Kruglik, and R. J. Dwayne Miller, Phys. Rev. Lett. 100, 155504 (2008).

[16] B. J. Siwick, J. R. Dwyer, R. E. Jordan, and R. J. D. Miller, Science 302, 1382 (2003).

[17] M. Z. Mo et al., Science 360, 1451 (2018).

[18] Z. Chen, M. Mo, L. Soulard, V. Recoules, P. Hering, Y. Y. Tsui, S. H. Glenzer, and A. Ng, Phys. Rev. Lett. 121, 075002 (2018).

[19] E. Bévillon, J. P. Colombier, V. Recoules, and R. Stoian, Phys. Rev. B 89, 115117 (2014).
[20] S. L. Daraszewicz, Y. Giret, N. Naruse, Y. Murooka, J. Yang, D. M. Duffy, A. L. Shluger, and K. Tanimura, Phys. Rev. B 88, 184101 (2013).

[21] S. I. Anisimov, B. L. Kapeliovich, and T. L. Perel'man, Sov. Phys. JETP 39, 375 (1974).

[22] Z. Lin, L. V. Zhigilei, and V. Celli, Phys. Rev. B 77, 075133 (2008).

[23] C. Guo, G. Rodriguez, A. Lobad, and A. J. Taylor, Phys. Rev. Lett. 84, 4493 (2000).

[24] M. Kandyla, T. Shih, and E. Mazur, Phys. Rev. B 75, 214107 (2007).

[25] Y. Ping, D. Hanson, I. Koslow, T. Ogitsu, D. Prendergast, E. Schwegler, G. Collins, and A. Ng, Phys. Rev. Lett. 96, 255003 (2006).

[26] K. Widmann, T. Ao, M. E. Foord, D. F. Price, A. D. Ellis, P. T. Springer, and A. Ng, Phys. Rev. Lett. 92, 125002 (2004).

[27] Z. Chen, B. Holst, S. E. Kirkwood, V. Sametoglu, M. Reid, Y. Y. Tsui, V. Recoules, and A. Ng, Phys. Rev. Lett. 110, 135001 (2013).

[28] T. Ao, Y. Ping, K. Widmann, D. F. Price, E. Lee, H. Tam, P. T. Springer, and A. Ng, Phys. Rev. Lett. 96, 055001 (2006).

[29] S. Mazevet, J. Clérouin, V. Recoules, P. M. Anglade, and G. Zerah, Phys. Rev. Lett. 95, 085002 (2005).

[30] B. I. Cho et al., Phys. Rev. Lett. 106, 167601 (2011).

[31] F. Dorchies et al., Phys. Rev. Lett. 107, 245006 (2011).

[32] B. Mahieu, N. Jourdain, K. Ta Phuoc, F. Dorchies, J-P. Goddet, A. Lifschitz, P. Renaudin, and L. Lecherbourg, Nat. Commun. 9, 3276 (2018).

[33] B. I. Cho, T. Ogitsu, K. Engelhorn, A. A. Correa, Y. Ping, J. W. Lee, L. J. Bae, D. Prendergast, R. W. Falcone, and P. A. Heimann, Sci. Rep. 6, 18843 (2016).

[34] N. Jourdain, L. Lecherbourg, V. Recoules, P. Renaudin, and F. Dorchies, Phys. Rev. B 97, 075148 (2018).

[35] P. M. Leguay, A. Lévy, B. Chimier, F. Deneuville, D. Descamps, C. Fourment et al., Phys. Rev. Lett. 111, 245004 (2013).

[36] V. Recoules and S. Mazevet, Phys. Rev. B 80, 064110 (2009).

[37] L. van Hove, Phys. Rev. 89, 1189 (1953).

[38] H. Ebert, J. Stöhr, S. S. P. Parkin, M. Samant, and A. Nilsson, Phys. Rev. B 53, 16067 (1996).

[39] X-Ray Absorption: Principles, Applications, Techniques of EXAFS, SEXAFS, XANES, edited by D.C. Koningsberger and R. Prins (John Wiley \& Sons, New York, 1988).

[40] N. Jourdain, V. Recoules, L. Lecherbourg, P. Renaudin, and F. Dorchies, Phys. Rev. B 101, 125127 (2020).

[41] F. Dorchies, N. Fedorov, and L. Lecherbourg, Rev. Sci. Instrum. 86, 073106 (2015).

[42] F. Dorchies, N. Jourdain, L. Lecherbourg, and P. Renaudin, Phys. Rev. E 98, 033212 (2018).

[43] See Supplemental Material at http://link.aps.org/supplemental/ 10.1103/PhysRevLett.126.065001 for more details.

[44] J.-P. Colombier, P. Combis, F. Bonneau, R. Le Harzic, and E. Audouard, Phys. Rev. B 71, 165406 (2005).

[45] A. V. Bushman, I. V. Lomonosov, and V. E. Fortov, Sov. Tech. Rev. B Therm. Phys. 5, 1 (1993).

[46] D. V. Minakov and P. R. Levashov, Phys. Rev. B 92, 224102 (2015)

[47] N. A. Smirnov, Phys. Rev. B 101, 094103 (2020).

[48] J. Gaudin et al., Sci. Rep. 4, 4724 (2014).

[49] B. Kettle et al., Phys. Rev. Lett. 123, 254801 (2019). 